\title{
Simple robust compensator for an adaptive optics system
}

\author{
Reziwan Maimaiti ${ }^{1, a}$ and Alimujiang Yiming ${ }^{2, b}$ \\ ${ }^{1}$ School of Electrical Engineering, Xinjiang University, Urumqi, Xinjiang, China \\ ${ }^{2}$ School of Electrical Engineering, Xinjiang University, Urumqi, Xinjiang, China \\ ammtreziwan@xju.edu.cn, balm_ym@xju.edu.cn
}

\begin{abstract}
Keywords: adaptive optics; robust control; reduced order; compensator; robustness
\end{abstract}
\begin{abstract}
To achieve a high-resolution imaging of targets in space, it is important to reduce the effects of atmospheric turbulence in adaptive optics systems. In this paper, we propose a simple and tunable low-order robust compensator for adaptive optics system, based on the robust model matching method. We have made some laboratory experiments with the atmospheric simulation instruments. Simulation and experimental results shows that the system with the designed controller has improved significantly comparing with the previous work.
\end{abstract}

\section{Introduction}

The earth's atmosphere introduces aberrations to the wavefront from an astronomical object when observed by a telescope. The objective of an adaptive optics (AO) system in astronomy is to reduce, as much as possible, the effects of these aberrations in real-time, by adjusting the shape of a deformable mirror (DM) to remove the aberration using closed loop feedback control [1, 2]. Classical integral feedback of the measured wavefront works well provided in slow operating condition at the AO system. However, with increasing demand of the control performance, more improved model-based control strategy is needed, and a practical AO system should be optimized to realize its best possible performance.

Several optimal control methods are applied based on accurate mathematical model of the system using identification technique, and modern control strategy offers better results [3, 4], however, it tends to lead high-order complex controllers, and the real-time computational burden is a major obstacle for realization of these potential performances.

Reduced order robust control is a potential solution to overcome such problem. There has been much study about robust control system design, and among them, robust model matching (RMM) $[5,6]$ is a natural and flexible method to design and analysis of robust control systems.

In this paper, we have designed a simple and tunable low-order robust compensator for an adaptive optics system, based on the RMM strategy. The major point of our work comparing with previous studies is that the design procedure is very simple and yields low-order controller, therefore, there is less real-time computational burden than high-order complex controllers. And then, because the compensator design is rather flexible, robustness can be tuned easily.

\section{Modeling structure of AO system}

Generally, an AO system consists of three main parts: a sensor referred to as the wavefront sensor (WFS), a compensator referred to as the control computer (CC), and a compensating device referred to as the deformable mirror (DM). The WFS determines the phase of an optical wavefront and is typically made up of a charge-coupled device (CCD) camera and specialized software that calculates the wavefront estimates. The CC calculates the corrections to be made and is the heart of the system. Finally, the deformable mirror implements the corrections.

As for the AO system is a standard closed-loop type, its simplified closed-loop block diagram can be described as Fig. 1, where CC is acted as a pure integrator, DAC is the digital-to-analog converter and its transfer function is denoted and approximated as $e^{-T_{d} s}$. The WFS is a real-time computer that reads and digitizes the detector signals, and applies specific algorithms to derive the wave-front measurements. Its transfer function can be approximated as unity because the main 
temporal characteristic of it is a pure time delay due to the read-out of the detector and the computation. HVA is the high voltage amplifier, it amplify the low voltage outputs of the DAC to drive the actuators of the DM. Its transfer function is close to unity. The DM temporal behavior is given by its mechanical response, characterized by resonance frequencies and damping factors. Its transfer function is considered as second order according to reference [1].

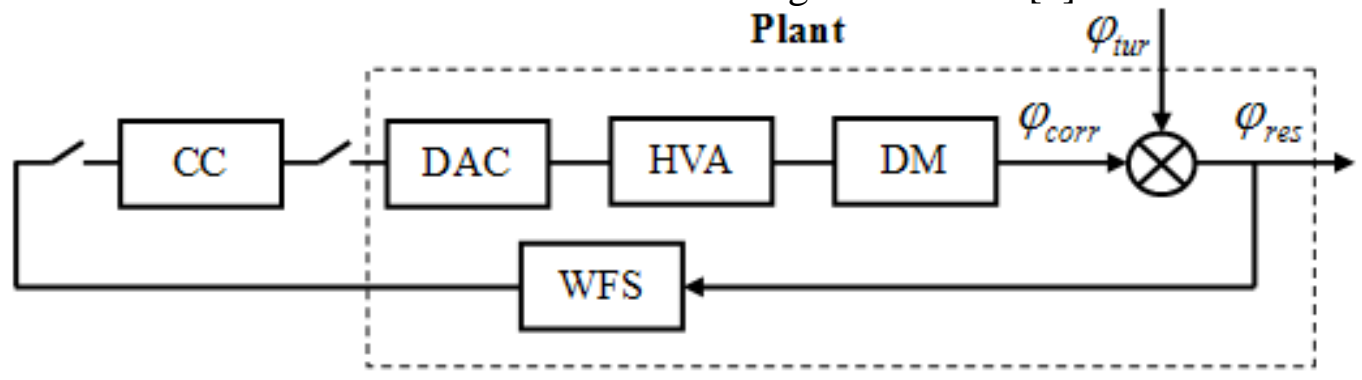

Figure. 1 Block diagram of the conventional AO system with integrator controller

As shown in the figure, our AO system is a feedback loop that involves both discrete-time and continuous-time signals. The control commands (computer's output) and measurements (computer's input) are discrete-time signals, and the others are continuous-time signals. Thus the structure of the feedback loop is a standard sampled-data system. The input of the system is the uncompensated wavefront whose value at a $2 \mathrm{D}$ (horizontal and vertical) point $(x, y)$ and a time $t$ is denoted by the $\varphi_{\text {tur }}(x, y, t)$. The correction introduced by the DM is denoted by the $\varphi_{\text {corr }}(x, y, t)$. The $\varphi_{\text {res }}(x, y, t)$ is the value of the residual phase after correction, so that

$$
\varphi_{\text {res }}(x, y, t)=\varphi_{\text {tur }}(x, y, t)+\varphi_{\text {corr }}(x, y, t)
$$

The purpose of the AO control system is to eliminate the effects of the disturbance $\varphi_{\text {tur }}(x, y, t)$, thus making the residual wavefront $\varphi_{\text {res }}(x, y, t)$ as small as possible. The AO system uses the WFS measured (ideally flat) residual wavefront to estimate the aberration induced by the turbulence, and to adjust the shape of the DM.

In order to obtain the accurate plant modeling structure of the AO system, we conducted the laboratory experiments. There are two driving modes for the mirror: tip-tilt and turbulence mode. We obtained the input and output data in the tip-tilt mode, and estimated the plant modeling structure using System Identification Toolbox in MATLAB, by applying the experimental input and output data. The estimated plant model $G(s)$ which is composed of DAC, HVA, DM and WFS in Fig. 1 is described by a second-order system $G_{1}(s)$ accompanied by a delay $G_{2}(s)$ as shown in the following equation:

$$
G(s)=G_{1}(s) \times G_{2}(s)=\frac{\omega_{n}{ }^{2}}{s^{2}+2 \zeta \omega_{n} s+\omega_{n}{ }^{2}} \times e^{-T_{d} s}
$$

where $\omega_{n}=3098, \zeta=0.626$, and $T_{d}=0.001299$

Fig. 2 shows the plant modeling structure estimation. The solid curve is the experimental output while the dotted curve represented the experimental input, and the dashed curve shows the estimated output by applying System Identification Toolbox. During the modeling structure estimation, the best fit between the experimental output and estimated output is more than $98 \%$. 


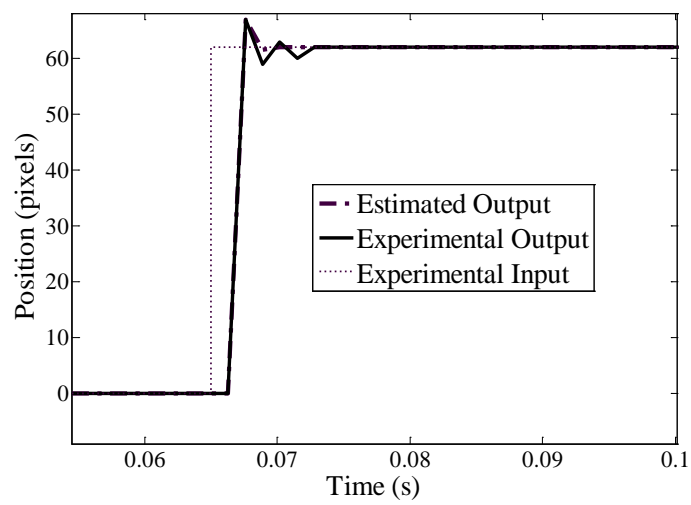

Fig. 2 Plant modeling structure estimation

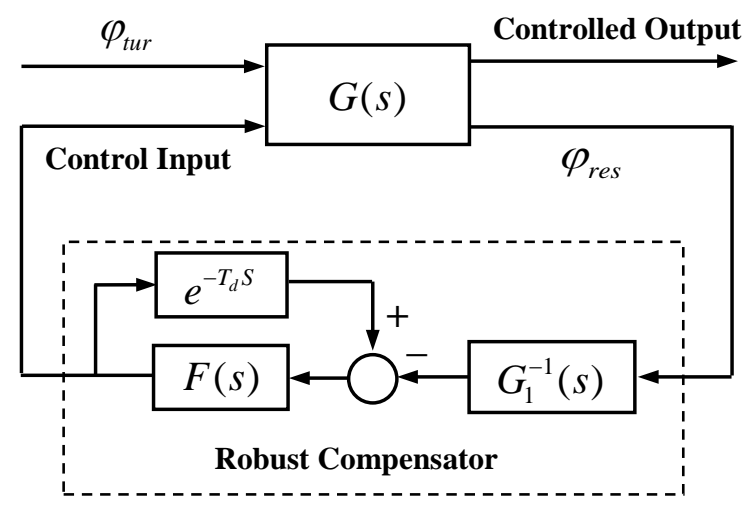

Fig. 3 Construction of robust control system

\section{Robust Compensator Design}

The controller of the previous experimental AO system is a pure integrator, therefore, the efficient frequency domain of the system performance is not as well as predicted. In order to modify the system performance and to improve the system robustness, it is necessary to apply more sophisticated techniques to design the controller. Robustness is an important subject for practical system designs based on model-based strategy. For practical control system design, both the performance and the configuration of the control system are important considerations. Parallel-model-and-plant structure is explicitly considered in several control strategies because the compensators design procedures are simple and practical. However, they require corresponding transfer functions of existing control systems. In our present work, we have designed a robust compensator based on the RMM strategy and modified the formulation of the RMM. Compared to the prior RMM and plug-in compensators that handle with closed-loop transfer functions including existing controller, the proposed method focuses on only transfer functions of the plants. Since this policy works any control systems, and the compensator is obtained without using transfer functions on previously designed feedback systems, it is applicable to any existing control systems. The structure of robust control system is shown in Fig. 3. The robust compensator consists of the Observer of equivalent disturbances and robust filter. The observer calculates equivalent disturbance from measured output ${ }_{\text {res }}$, and cancels the effect of plant's changes by minimizing transfer function of overall system from equivalent disturbance to measured output. In order to execute disturbance rejection with the robust stability, a transfer function called robust filter $F(s)=1000 /(s+1000)$ is used.

To specify the frequency response characteristics of the robust control system, we made the bode plot and compared with the previous system. Fig. 4(a) is the open-loop bode plot of the system using pure integrator, and Fig. 4(b) give the open-loop bode plot of the system using the robust compensator. We compared the bandwidths for both robust compensator and pure integrator systems. As for the $0 \mathrm{~dB}$ open-loop cut-off frequency, the robust control system have better frequency domain comparing with the previous pure integrator system. 


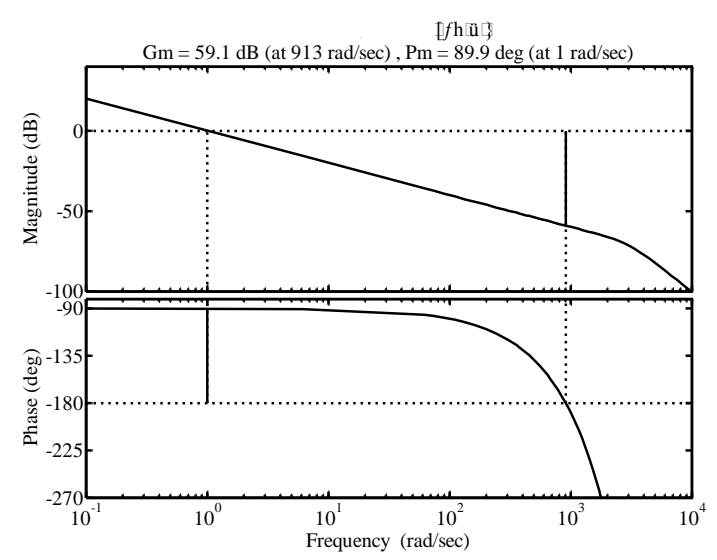

(a) System using pure integrator

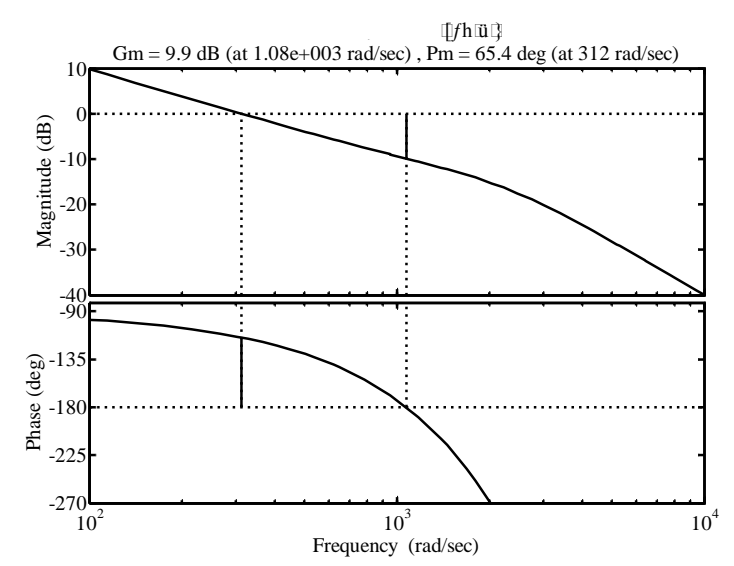

(b) System using robust compensator

Fig. 4 Frequency response characteristics of the systems

Another factor for the bandwidth is the $0 \mathrm{~dB}$ closed-loop error cut-off frequency. This definition also is of great interest since it is related to the residual optical phase after correction. Both the bandwidths of the open-loop 0dB frequency and the closed-loop error cut-off frequency are defining the real performances of the system where the AO system is efficient. As shown in Fig. 5, one curve is the $0 \mathrm{~dB}$ closed-loop error cut-off frequency for the system using pure integrator and the value of the bandwidth is $2.8 \mathrm{~Hz}$; and the another curve is the $0 \mathrm{~dB}$ closed-loop error cut-off frequency for the system using robust compensator and the value of the bandwidth is about $50 \mathrm{~Hz}$. From the figure we can also learn that the robust control system have better frequency domain comparing with the previous pure integrator system.

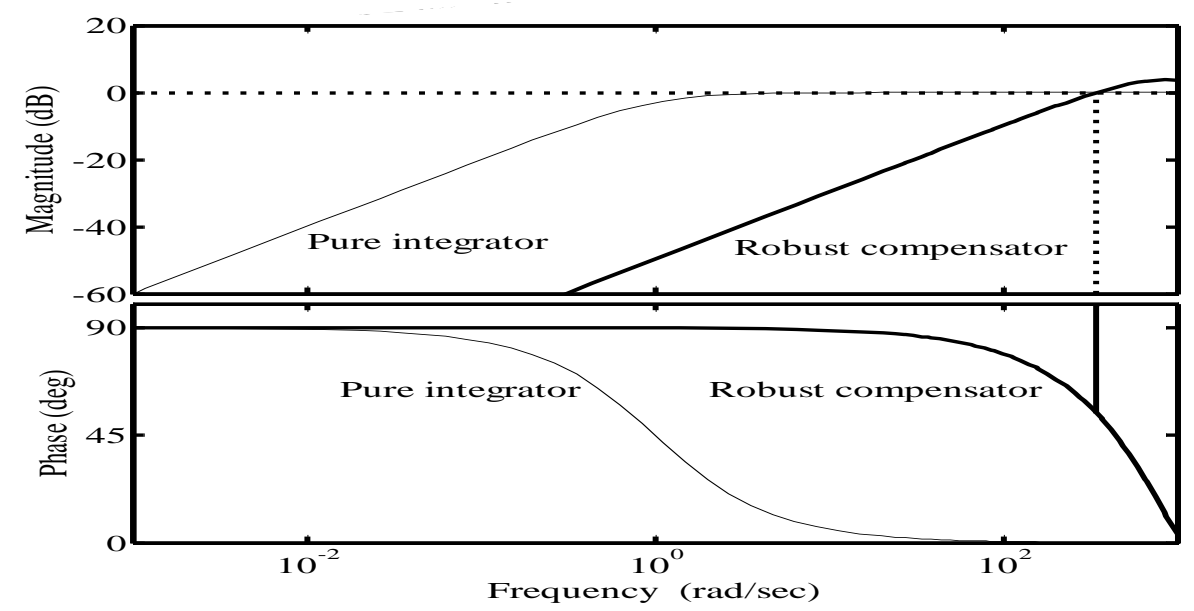

Fig. 5 Gain of the closed-loop error cut-off frequency

Also, In order to verify the robustness of the system, we introduced some noises and made simulation in MATLAB. As shown in Fig. 6, the Band-Limited white noise acted as uncompensated wavefront, Fig. 6(a) and 6(b) show the values of the residual phase after reducing the Band-Limited white noise, by using pure integrator and robust compensator respectively. As shown in the figures, the value of the residual phase is tending to be minimized instantly by using robust compensator, comparing to the use of pure integrator. 


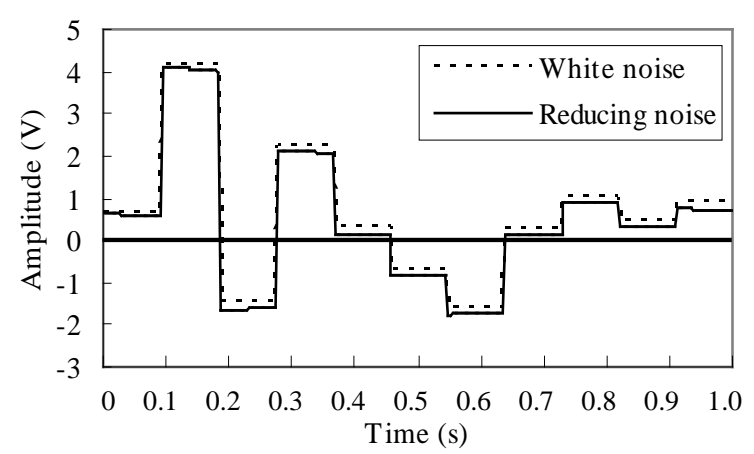

(a) Using pure integrator

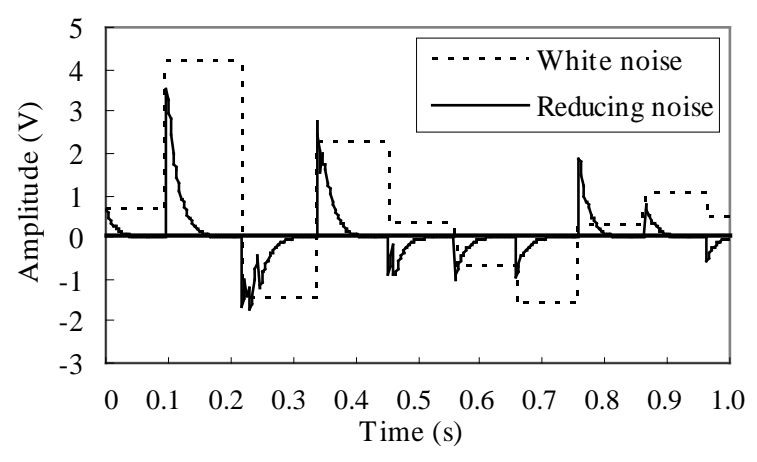

(b) Using robust compensator

Fig. 6 Simulation results for comparison of reducing the noise

\section{Experiment and result analyzing}

In order to investigate the performance of the AO system, the laboratory experiments were conducted. To simulate atmospheric turbulence, we introduced another deformable mirror that is a membrane type with 19 channels. As mentioned in Section 2, there are two driving modes for the mirror: tip-tilt and turbulence. In the tip-tilt mode, the mirror surface remains flat but inclines with a given oscillation frequency. When the turbulence mode is selected, the mirror surface is corrugated with a given frequency. These frequencies can be tuned by a parameter in the software.

In our experiment, we set the oscillator frequency in the tip-tilt mode and changed the tip-tilt frequency. Table 1 listed the results of 32, 64, 99, 130 and $172 \mathrm{~Hz}$. From the table we can see that with applying the AO system, by the frequency of 32, 64 and $99 \mathrm{~Hz}$, the deviations both in horizontal and vertical directions were improved significantly using the robust compensator comparing to the use of pure integrator. As the driving frequency increased, the results are no more improved comparing to the use of pure integrator. We consider that one of the main reasons is the insufficiency of the processing speed as the driving frequency increased, because the whole AO system is a software-oriented system.

Table 1. Deviations of centroid positions.

\begin{tabular}{|c|c|c|c|c|c|c|}
\hline \multirow{2}{*}{$\begin{array}{c}\text { Oscillation } \\
\text { frequency } \\
\text { (Hz) }\end{array}$} & \multicolumn{2}{|c|}{ Without AO } & \multicolumn{2}{c|}{$\begin{array}{c}\text { With AO } \\
\text { (pure integrator) }\end{array}$} & \multicolumn{2}{c|}{$\begin{array}{c}\text { With AO } \\
\text { (robust compensator) }\end{array}$} \\
\cline { 2 - 7 } & $\begin{array}{c}\text { Horizontal } \\
\text { (pixels) }\end{array}$ & $\begin{array}{c}\text { Vertical } \\
\text { (pixels) }\end{array}$ & $\begin{array}{c}\text { Horizontal } \\
\text { (pixels) }\end{array}$ & $\begin{array}{c}\text { Vertical } \\
\text { (pixels) }\end{array}$ & $\begin{array}{c}\text { Horizontal } \\
\text { (pixels) }\end{array}$ & $\begin{array}{c}\text { Vertical } \\
\text { (pixels) }\end{array}$ \\
\hline 32 & 3.519353 & 0.169163 & 3.421988 & 0.154648 & 1.713947 & 0.128598 \\
\hline 64 & & & 3.455884 & 0.155137 & 3.283632 & 0.142491 \\
\hline 99 & & & 3.496365 & 0.150535 & 3.452074 & 0.145023 \\
\hline 130 & & & 3.496584 & 0.153162 & 3.836154 & 0.171719 \\
\hline 172 & & & 3.497399 & 0.153812 & 3.908771 & 0.138894 \\
\hline
\end{tabular}

\section{Summary}

In this paper, we have designed a simple and practical robust compensator for an AO system, based on the RMM strategy and modified the formulation of the RMM. Because the design procedure is simple and yields low-order controllers, there is less real-time computational burden than high-order complex controllers.

To investigate the robustness and the performance of the AO system, we made MATLAB simulations as well as laboratory experimentations. Simulation and experimental results shows that the system with the robust controller has improved significantly comparing to the previous work. 
For the future work, the processing speed of the AO system should be improved in order to overcome the insufficiency of the processing speed. Moreover, adjusting the parameters of the robust compensator also is our further consideration.

\section{Acknowledgment}

This work is financially supported by National Natural Science Foundation of China (61263009).

\section{References}

[1] J. W. Hardy, Adaptive optics for astronomical telescopes, New York: Oxford Univ. Press (1998).

[2] R. Typson, Principles for adaptive optics, 2nd ed., New York: Academic Press (1998).

[3] J. Huang, D. P. Looze, N. Denis, and D. A. Castanon, in: Control Designs for an Adaptive Optics System, Proceedings of the $34^{\text {th }}$ Conference on Decision \& Control, New Orleans, LA, December (1995), p. 3753-3756

[4] B. W. Frazier and R. K. Tyson, in: Robust Control of an Adaptive Optics System, Proceedings of the Thirty-Fourth Southeastern Symposium on System Theory (2002).

[5] T. Eisaka, Y. Zhong, S. Bai and R. Tagawa, in: Evaluation of robust model matching for the control of a DC servo motor, International Journal of Control, Vol. 5 (1989), p. 479-493.

[6] T. Eisaka, and W. Xie, in: Design of Add-on Robust Compensators for any Existing Controllers, SICE 2004 Annual Conference, Vol. 3 (2004), p. 2291-2297. 